\title{
Stress and cortisol as predictors of fatigue in medical/surgical nurses and nurse leaders: A biobehavioral approach
}

\author{
Mona Cockerham ${ }^{* 1,2}$, Duck-Hee Kang ${ }^{3}$, Robin Howe ${ }^{2}$, Susan Weimer ${ }^{1}$, Lisa Boss ${ }^{4}$, Sharvari R. Kamat ${ }^{3}$ \\ ${ }^{1}$ Sam Houston State University, Huntsville, Texas, United States \\ ${ }^{2}$ Houston Methodist Willowbrook Hospital, Houston, Texas, United States \\ ${ }^{3}$ School of Nursing, University of Texas Health Science Center, Houston, Texas, United States \\ ${ }^{4}$ Memorial Hermann, Southwest, Houston, Texas, United States
}

Received: July 6, 2017

DOI: $10.5430 /$ jnep.v8n5p76
Accepted: August 7, 2017

Online Published: December 27, 2017

\begin{abstract}
Objective: High acuity and long work hours are significant contributors to nurses' stress. Studies evaluating consecutive workdays with the use of biobehavioral methods are limited in the US. The aim of this study was to assess changes in and the relationship between stress, fatigue, and cortisol.

Methods: In an observational within-subject design, we studied stress, fatigue and cortisol before and after 2 consecutive 12 -hour day shifts in an acute care setting. Specifically, the study was designed to: (1) assess the effect of stress on fatigue; (2) examine the effect of stress on cortisol; (3) compare the levels of stress, fatigue, and cortisol; and (4) compare the responses of stress, fatigue, and cortisol between acute care, day shift staff nurses and nurse leaders.

Results: Stress, fatigue, and cortisol increased significantly from baseline to Day 2 ( $p=.001, .004$, and .010 , respectively; paired $t$-test). In a comparison of nurses and nurse leaders, stress and fatigue at baseline were significantly higher in acute care nurses than in nurse leaders ( $p \geq .00$ and .05 , respectively; independent $t$-test). At the end of 2 consecutive shifts, cortisol was significantly higher in staff nurses than in nurse leaders $(p=.001)$.

Conclusions: Competing initiatives pressure nurse leaders to work long hours to support organizational goals, sometimes at the expense of a healthy work environment. Nurses from direct care staff to executives should be educated in and demonstrate best practices in relation to endorsements from the American Nurses Association on fatigue and interventions to lessen the risks to patient safety.
\end{abstract}

Key Words: Cortisol, Fatigue, Stress, Medical/Surgical, Nurses, Leaders

\section{INTRODUCTION}

Long hours with constant interruptions, complex patient care needs, and draining emotional interactions with patients and families contribute to work-related stress for nurses. Workrelated stress was reported as the number one perceived work hazard that places nurses at risk of harm in high-acuity ar- eas such as critical and emergency care areas. ${ }^{[1]}$ Stress is associated with a variety of cognitive functions, physical injuries, and practice errors that lead to fatigue and patient errors. ${ }^{[2,3]}$ Evidence supports that overtime hours have potential risk to patient safety related to adverse events and errors in care. ${ }^{[4]}$ Working a nursing job requiring on-call

*Correspondence: Mona Cockerham; Email: mcc053@shsu.edu; Address: Sam Houston State University, Huntsville, Texas, United States. 
hours leads to overtime, and limits the ability for the nurse to control the number of hours worked, and the ability to detach from work leading to fatigue and sleep disturbances. ${ }^{[5]}$ Kang et al. ${ }^{[6]}$ recommend that the effects of stress be evaluated by the use of biobehavioral methodologies beyond the use of self-reporting instruments.

Cortisol is a hormone produced by the adrenal glands that affects metabolism and the regulation of the immune system. Imbalances in cortisol levels can lead to fatigue, depression, obesity, and immune dysfunction. ${ }^{[7,8]}$ Stress leads to adverse physical health through a biological response in the body as the result of hypercortisolemia and the inflammatory response. ${ }^{[8]}$ Stress activates a physiological response in which the hypothalamus-pituitary-adrenal (HPA) axis acts through hypercortisolemia, increasing circulating cortisol in the blood. ${ }^{[7]}$ Increased cortisol levels inhibit insulin secretion and muscle glucose absorption. The density of cortisol receptors in the abdomen leads to the uptake of excessive cortisol in the inter-abdominal fat causing abdominal obesity. ${ }^{[7]}$ In addition, the inflammatory response caused by the release of cytokines can interfere with insulin signaling and lead to insulin resistance, although this is not measured in this study.

Fatigue is often acute, encompasses physiological and psychological symptoms, and manifests as behaviors from tiredness to exhaustion and can lead to decrements in cognitive functioning and decreased performance. Moreover, unresolved fatigue can progress to chronic fatigue, depression, and burnout, influencing work performance and potentially affecting patient care. ${ }^{[9]}$ Chen et al. ${ }^{[10]}$ reported that fatigue may lead to increased errors, absenteeism, turnover, and reduced productivity. The cost of job-related injuries for nurses is estimated to exceed $\$ 200$ billion a year. ${ }^{[11]}$ In a survey of over 3,000 Registered Nurses by the American Nurses Association (ANA) and the Health Resources and Services Administration (HRSA), 60\% of nurses reported having worked through their breaks, having come in early, or having worked late to complete their work; one-third perceived workloads to be high; and $82 \%$ identified work-related stress as the top work health and safety risk. ${ }^{[12]}$ Research evidence suggests that shift work, working long hours, consecutive work days, and accumulation of sleep debt increases fatigue. ${ }^{[2,3,13-15]}$ Higher levels of fatigue have been reported on night shift than on day shift by nurses. ${ }^{[16]}$ In an integrative review evaluating fatigue in acute care settings, researchers found that shifts longer than 12 hours contribute to increased fatigue. ${ }^{[1]}$ According to the US Department of Health and Human Services, $75 \%$ of nurses in US hospitals work 12hour plus shifts. ${ }^{[17]}$ There are many reasons nurses choose to work 12-hour shifts, which is often to increase work-life balance, but at times at the expense of sleep. ${ }^{[5]}$ Overtime

Published by Sciedu Press has been a prevalent practice as the result of chronic understaffing and times of high census related to winter months. ${ }^{[14]}$ Overtime can increase the number of incidents of needlestick injuries, musculoskeletal injuries, and illnesses. ${ }^{[18]}$ Few studies, however, have evaluated the association of stress, fatigue, and cortisol over consecutive shifts.

\subsection{Purpose of this study}

The primary purpose of this study was to measure changes in perceived stress, fatigue, and salivary cortisol levels in medical/surgical staff nurses and nurse leaders working 2 consecutive 12-hour shifts in an acute care setting. The secondary purpose was to compare levels of perceived stress and work-related stress, fatigue, and salivary cortisol between staff nurses and nurse leaders. The hypotheses were as follows: (1) stress and fatigue will be significantly higher at the end of a 12-hour shift on Day 2 than at the beginning of Day 1, (2) AM and PM cortisol levels will be higher on Day 2 than on Day 1, and (3) stress, fatigue, and cortisol will be higher in medical/surgical staff nurses than in nurse leaders.

\subsection{Conceptual framework}

Few researchers have evaluated the effects of stress in nurses working consecutive 12-hour workdays, particularly from a biobehavioral perspective. The use of biobehavioral approaches has been highly recommended by the National Institute of Nursing Research. ${ }^{[19]}$ The conceptual framework of the present study was based on the Expanded Biobehavioral Interactions Model in which various factors within personal, environmental, psychosocial, and behavioral domains affect biological responses and health outcomes. ${ }^{[6]}$ This framework provides a multidimensional and holistic view of how various antecedents of an individual's psychosocial, environmental, and behavioral domains influence biological responses of the immune system, central nervous system, and health outcomes. In this study, we focused on the relationships among stress (psychosocial domain), the stress hormone cortisol (biological domain), and fatigue (health outcome).

\subsection{Literature review}

In 2 studies conducted in Taiwan, researchers reported that the fast-forward rotating shift is common in Taiwan, which includes 8-hour rotating shifts of 2 days, 2 evenings, and 2 nights. One study evaluated performance on various instruments and found few negative effects and reported that cortisol levels decreased during the morning following the last shift. ${ }^{[20]}$ Another study found that nurses who worked 2 consecutive rotating 8-hour shifts had higher anxiety (stress) and poorer performance than did those who worked 4 consecutive 8-hour shifts; however, the sample size was small. ${ }^{[21]}$ Chen et al. ${ }^{[10]}$ collected data on a very small number of 
nurses during 2 consecutive 12-hour shifts and reported heart rate elevations associated with increased acute fatigue with care activities such as manual patient handing versus care coordination activities. ${ }^{[10]}$ In a study of nurses working 3 consecutive 12-hour days, nights, fixed, or rotating shifts, Han et al. ${ }^{[2]}$ reported that nurses working rotating shifts had higher levels of acute fatigue than did those on days, nights, or fixed shifts. In addition, that study did not report a significant difference between day and night shift nurses related to acute or chronic fatigue. Lin et al. ${ }^{[23]}$ reported associations on work-related (occupational) stress of working consecutive 8-hour rotating shifts on days, evenings, nights, or rotating shifts. Using an effort and reward instrument, the authors found that nurses on rotating shifts, or nurses working 7 consecutive shifts, reported significantly higher stress scores than did nurses who worked day or night shifts. ${ }^{[23]}$ That study also reported that having at least 2 days off can reduce the effects of stress. Geiger-Brown et al. ${ }^{[2]}$ reported that nurses acquire significant sleep debt while working 3 consecutive 12-hour shifts, with $36 \%$ of nurses reporting a high level of baseline and inter-shift fatigue. Nui et al. ${ }^{[24]}$ reported in a systematic review of shift rotations, cortisol levels, and fatigue that night shift nurses experience interruptions in circadian rhythms leading to disruptions in sleep periods, feelings of malaise, and decreased mental alertness due to increased cortisol secretions during the daytime while sleeping. This study compared effects between nurses working day and night shifts.

Stress biomarkers aid in detecting early signs of developing negative health outcomes. ${ }^{[25]}$ Cortisol hormone is a biomarker released in response to stress that is considered to be an objective measurement. ${ }^{[26]}$ Under stress, activation of the HPA axis releases hormones that signal the adrenal cortex to produce cortisol. ${ }^{[7]}$

\section{Methodology}

\subsection{Sample/setting}

Of the 70 day-shift medical-surgical nurses recruited, data from 11 nurses were missing owing to unexpected schedule changes, attrition, or insufficient saliva samples for cortisol assessment. Of the remaining 59 nurses, 16 were dayshift full-time nurse leaders either managers or directors and 43 were medical/surgical staff nurses at a 313 bed Magnetdesignated suburban hospital. The staff nurses and leaders were scheduled to work 12-hour shifts. The nurse patient ratio was $1 \mathrm{RN}$ to 4-5 patients for staff nurses with limited support staff and responsible for patient transport and all laboratory specimen draws. The nurse leaders were responsible for 20-36 bed hospital unit. Power analysis indicated a minimum sample size of 60 to meet the power level of
$80 \%$ and alpha level of .05, assuming moderate correlation between stress and cortisol. Participation was voluntary and uncompensated.

\subsection{Recruitment}

Invitation letters were sent to 250 nurses (RNs) and nurse leaders (managers $\&$ directors) in medical/surgical acute care units (adult patients), and fliers were distributed at one hospital. Interested participants signed a consent form. Inclusion criteria were as follows: (1) full-time nurses who had worked within the 2 weeks prior to the study, so as not to allow certain nurses more rest to control for the potential effects of vacation in lowering stress and fatigue levels prior to the beginning of the study; and (2) nurses who were scheduled to work a 12-hour day or night shift for 3 or more consecutive days. Excluded were nurses (1) working fewer than 36 hours per week, (2) using steroids or anti-inflammatory medications within the last 2 weeks, and/or (3) with a current viral or bacterial infection. The study was approved by the university and hospital institutional review boards. Subjects were assigned a code number for data collection and anonymity was guaranteed.

\subsection{Instruments and saliva collection}

Instruments used for collecting self-reporting data were as follows:

(1) Multidimensional Fatigue Inventory (MFI-20). This widely used, 20-item instrument contains 4 subscales: perceived general, physical, and mental fatigue along with reduced activity and motivation. The tool uses a 5-point Likert scale, where $1=$ agree to $5=$ disagree. Higher scores indicate higher fatigue. Testretest reliability coefficients range from .74 to .87 , and Cronbach's $\alpha$ ranges from $.85 .96 .^{[27]}$ In this study, Cronbach's $\alpha$ was 0.86 .

(2) Nursing Stress Scale (NSS). This well-known instrument has 11 subscales associated with general working conditions to specific stressors in hospital nursing, plus an overall stress score. The instrument uses a 4-point Likert scale, where $1=$ no stress to $4=$ extreme stress. Cronbach's $\alpha$ ranges from .66 to .87, and the test-retest coefficient for the total scale is $.81 .^{[28]}$ In this study, Cronbach's $\alpha$ was 0.77 .

(3) Perceived Stress Scale (PSS). This widely used instrument provides a nonspecific and interpersonal appraisal of stress during the past month. The higher the score, the higher the perceived stress level. Cronbach's $\alpha$ is .86, and the test-retest reliability coefficient is .85. ${ }^{[29]}$ In this study, Cronbach's $\alpha$ was 0.84 .

(4) Visual Analogue Scale (VAS). The VAS-Stress (VAS$\mathrm{S})$ and VAS-Fatigue (VAS-F) scores range from 0 
(none) to 10 (high) and were used for repeated measures of stress and fatigue before and after shifts.

(5) Salivary cortisol was measured by using enzymelinked immunoassay kits (Salimetrics, PA). Saliva samples were collected as indicated below, transported in an ice-packed cooler to a laboratory, and stored in a 80 -degree freezer until batch assayed. The sensitivity for cortisol was $<0.003 \mu \mathrm{g} / \mathrm{dL}$, and the coefficients of variation for intra-assay and inter-assay precision were $5.97 \%$ and $13.97 \%$, respectively, for this study.

\subsection{Procedures}

Participants completed baseline demographic information and the questionnaires (PSS, NSS, MFI) before the start of their shift on Day 1. Saliva samples and VAS-S and VAS-F responses were collected at the beginning and end of each of the 2 consecutive 12-hour workdays (Day 1 and Day 2). The 4 data collection points were Day $1 \mathrm{AM}$, Day $1 \mathrm{PM}$, Day 2 AM, and Day 2 PM between 6:30 AM and 7:15 AM and for end of shift between 6:45 PM and 7:15 PM to control for circadian rhythmicity. Each nurse collected his or her own saliva samples using a passive drool method, documented the saliva collection time, and placed the samples in a refrigerator until transport to the laboratory.

\subsection{Statistical methods}

Data were checked for accuracy and completion and then transferred to SPSS 21 for statistical analysis. The associations between baseline questionnaire scores (PSS, NSS, MFI) and repeated-measures scores (VAS-S, VAS-F, cortisol) were assessed at 4 time points. Paired comparison $t$-tests were used to evaluate the overall change in stress and fatigue from Day 1 AM to Day 2 PM. Cortisol was evaluated at all 4 time points to evaluate for changes in cortisol from the beginning to the end of a shift. Univariate and multivariable predictors of VAS-F and MFI-20 were assessed by using linear regression. Differences in stress, fatigue, and cortisol between nurses and nurse leaders were evaluated at each time point by using independent sample $t$-tests. Cortisol values did not require transformation because of the normality of the distribution. All $p$ values were two-sided and considered significant when less than .05 .

\section{Results}

\subsection{Sample characteristics}

Participants were predominantly female nurses ( $n=61$ of 70). The mean age of the nurses in this study was 39.5 years. This study represented experienced nurses with a mean of 16.8 years of experience. The majority of nurses (78\%) had a BSN degree. Hours worked per day and fatigue scores

Published by Sciedu Press were similar between the groups, whereas RN stress scores were higher in staff nurses than in nurse leaders (see Table 1). Overall perceived stress scores were higher in nurse leaders, but not significantly so. Nurse leaders, compared with medi$\mathrm{cal} /$ surgical nurses, reported greater fatigue (mean $=45.73$ to $43.79, p=.05$ ), less work-related stress (mean $=54.29$ to $81.26, p=.002)$, and more interpersonal stress (mean $=16.33$ to $15.62, p=.06$ ). However, at baseline, nurse leaders scored lower on the VAS-S (mean $=3.50$ ) than did medical/surgical nurses $($ mean $=5.03 ; p=.003)$.

Table 1. Demographic variables and fatigue and stress scores of nurse leaders and staff nurses at baseline

\begin{tabular}{lll}
\hline \multirow{2}{*}{ Demographic Variable } & Nurse Leaders & Staff Nurses \\
\cline { 2 - 3 } & Mean (SD) & Mean (SD) \\
\hline Age, years & $44(11.44)$ & $35(10.9)$ \\
Education* & $3.50(0.610)^{* *}$ & $2.9(0.52)^{* *}$ \\
Years of Experience & $20.89(10.15)$ & $12.8(7.98)^{* *}$ \\
Hours Worked per Week & $50.28(6.53)$ & $42.9(5.63)$ \\
Hours Worked per Day & $12.33(0.767)$ & $12.75(1.16)$ \\
Hours of Sleep per Night & $6.44(1.09)$ & $6.20(1.11)$ \\
MFI Score & $45.73(12.73)$ & $43.79(13.64)$ \\
RN Stress Score & $54.29(20.13)$ & $81.26(48.04)^{* *}$ \\
PSS Score & $16.33(5.40)$ & $15.62(6.57)$ \\
VAS-S Baseline & $3.50(2.74)$ & $5.03(2.44)$ \\
\hline Note. MFI: Multidimensional Fatigue Inventory; PSS: Perceived Stress Scale; \\
VAS-S: Visual Analogue Scale-Stress. \\
*2=ADN; $=$ BSN; $=$ MSN. ** $p \geq .005$.
\end{tabular}

\subsection{Descriptive data on stress, fatigue, and cortisol}

Mean scores for the combined groups at the baseline collection (Day $1 \mathrm{AM}$ ) and the other data collections points (Day 1 PM, Day 2 AM, and Day 2 PM) are shown in Table 2. Mean scores for repeated measures of stress and fatigue using the VAS instrument were relatively low, around 3 to 4 on a scale of 0 to 10 . This was interpreted as indicating low stress and fatigue. The baseline stress instruments (PSS and NSS) reported low to moderate levels of stress. The baseline multidimensional fatigue instrument (MFI-20) reported moderate levels of fatigue prior to starting 2 consecutive shifts (see Table 2).

\subsection{Comparison of stress, fatigue, and cortisol between day 1 and day 2}

Baseline stress, as measured by the PSS, NSS, and VAS-S instruments, was not a significant univariate or multivariate predictor of fatigue on Day 2. Stress and fatigue continued to increase from Day 1 to Day 2. Stress and fatigue were rated higher at the beginning than at the end of the shift. Cortisol decreased from morning to night on both Day 1 and Day 2 , which is a normal expectation for circadian rhythm. In a 
paired $t$-test, stress and fatigue were shown to significantly increase at the end of the shift on Day 2 compared with baseline at the beginning of the shift on Day 1, as shown in Table 3. Paired $t$-tests for cortisol were negative: cortisol levels for
Day 1 were higher than for Day 2. For cortisol, both morning and evening cortisol levels indicated a significant decrease on Day 2 from Day 1.

Table 2. Descriptive data on stress, fatigue, and cortisol

\begin{tabular}{llllll}
\hline Time point & Variable & N & Range & Mean & (SD) \\
\hline & VAS-Stress & 57 & $(0-10)$ & 3.13 & $(2.39)$ \\
& VAS-Fatigue & 57 & $(0-10)$ & 3.40 & $(2.81)$ \\
Day 1 AM & Cortisol & 58 & $(0.0257-0.9696)$ & 0.3101 & $(0.2223)$ \\
& MFI & 58 & $(21-78)$ & 44.48 & $(13.3)$ \\
& VAS-10 & 51 & $(0-10)$ & 4.75 & $(2.54)$ \\
& PSS & 59 & $(1-30)$ & 5.78 & $(6.31)$ \\
Day 1 PM & NSS & 59 & $(15-36)$ & 14.95 & $(44.9)$ \\
& Stress VAS & 58 & $(0-10)$ & 4.68 & $(2.70)$ \\
& Fatigue VAS & 58 & $(0-10)$ & 3.76 & $(2.70)$ \\
Day 2 AM & Cortisol & 57 & $(0000-0.4713)$ & 0.0860 & $(0.0968)$ \\
& Stress VAS & 56 & $(0-10)$ & 3.25 & $(2.50)$ \\
Day 2 PM & Fatigue VAS & 58 & $(0-10)$ & 3.76 & $(2.66)$ \\
& Cortisol & 57 & $(0000-0.8007)$ & 0.2055 & $(0.1535)$ \\
& Stress VAS & 59 & $(0-10)$ & 4.82 & $(2.70)$ \\
& Fatigue VAS & 58 & $(0-10)$ & 4.79 & $(2.57)$ \\
\end{tabular}

Note. MFI: Multidimensional Fatigue Inventory; NSS: Nursing Stress Scale; PSS: Perceived Stress Scale; VAS: Visual Analogue Scale-Stress and Fatigue. Cortisol was measured in $\mu \mathrm{g} / \mathrm{dL}$ by using the Salimetrics kit.

Table 3. Results of paired $t$-tests comparing stress, fatigue, and cortisol between Day 1 AM and Day 2 PM

\begin{tabular}{lllll}
\hline Variable 1 & Variable 2 & N & Mean Difference & p \\
\hline Day 2 PM_Stress & Day 1 AM_Stress & 54 & 3.46 & .001 \\
Day 2 PM_Fatigue & Day 1 AM_Fatigue & 53 & 3.54 & .004 \\
Day 2 PM_Cortisol & Day 1 AM_Cortisol & 52 & 0.238 & $.000^{*}$ \\
Day 2 AM_Cortisol & Day 1 AM_Cortisol & 54 & -0.109 & .001 \\
Day 2 PM_Cortisol & Day 1 PM_Cortisol & 53 & -0.038 & .010 \\
\hline
\end{tabular}

Note. Stress and fatigue were measured by Visual Analogue Scale. Cortisol was measured in $\mu \mathrm{g} / \mathrm{dL}$ by using the Salimetrics kit. *Less than .001.

3.4 Comparison between staff nurses and nurse leaders

Stress (VAS-S) and fatigue (VAS-F) at baseline were significantly higher for medical/surgical nurses than for nurse leaders on Day 1 AM $(p \geq .05)$. Likewise, cortisol levels were significantly higher for medical/surgical nurses ( $p=$ .001) than for nurse leaders on Day 2 PM (see Table 4). Otherwise, stress, fatigue, and cortisol responses were mostly similar between the 2 groups.

\section{Discussion}

Baseline stress as measured by the PSS, NSS, and MFI was not a predictor of fatigue at the end of the second-day con- secutive shift as hypothesized; only VAS-S $(p=.002)$ was a significant predictor of fatigue. A significant increase in stress and fatigue over 2 workdays supported the hypothesis that levels of stress and fatigue would be higher at the end of 2 consecutive 12-hour workdays. The decrease in cortisol from Day 1 to Day 2 did not support the hypothesis that cortisol increases over consecutive workdays. Cortisol was not significantly different between nurses and nurse leaders except at the end of Day 2, when the nurse leaders' cortisol scores increased to align closely with those of the nurses (see Table 4). The third hypothesis, that stress, fatigue, and cortisol would be higher in medical/surgical nurses than in nurse leaders at the end of 2 workdays, was supported. 
Table 4. Results of independent sample $t$-tests over four time-points between Day 1 AM and Day 2 PM for nurse leaders and staff nurses

\begin{tabular}{|c|c|c|c|c|c|}
\hline Time point & Variable & $\begin{array}{l}\text { Nurse Leaders } \\
(\mathrm{N}=16), \text { Mean (SD) }\end{array}$ & $\begin{array}{l}\text { Staff Nurses } \\
(\mathrm{N}=43), \text { Mean (SD) }\end{array}$ & Mean Difference & $p$ \\
\hline \multirow{3}{*}{ Day 1 AM } & Stress VAS & $1.2(1.03)$ & $3.3(2.01)$ & 2.060 & .000 \\
\hline & Fatigue VAS & $2.1(1.95)$ & $4(2.77)$ & 1.571 & .050 \\
\hline & Cortisol & $0.3394(0.2305)$ & $0.3180(0.2241)$ & -0.0214 & .779 \\
\hline \multirow{3}{*}{ Day 1 PM } & Stress VAS & $4.3(2.84)$ & $5(2.95)$ & 0.7555 & .418 \\
\hline & Fatigue VAS & $4.3(2.91)$ & $5(2.68)$ & 0.5557 & .550 \\
\hline & Cortisol & $0.1018(0.1460)$ & $0.0736(0.0514)$ & -0.0282 & .507 \\
\hline \multirow{3}{*}{ Day 2 AM } & Stress VAS & $2.6(2.60)$ & $4.5(2.35)$ & 0.8475 & .310 \\
\hline & Fatigue VAS & $3.6(2.99)$ & $3.8(2.63)$ & 0.2333 & .805 \\
\hline & Cortisol & $0.14(0.2186)$ & $0.41(0.2041)$ & -0.0144 & .769 \\
\hline \multirow{3}{*}{ Day 2 PM } & Stress VAS & $4.5(2.65)$ & $5(2.78)$ & 0.4239 & .613 \\
\hline & Fatigue VAS & $4(2.03)$ & $5(2.76)$ & 0.9571 & .175 \\
\hline & Cortisol & $0.0224(0.0275)$ & $0.0590(0.0446)$ & 0.0365 & .001 \\
\hline
\end{tabular}

Note. Cortisol was measured in $\mu \mathrm{g} / \mathrm{dL}$ by using the Salimetrics kit.

Cortisol exhibits a 24-hour diurnal pattern, with the maximum level reached prior to awakening and with a gradual decline throughout the day to the lowest levels during the night. ${ }^{[26]}$ We generalized that awakening was at least 1 hour earlier than the time set for collecting cortisol samples (6:307:30 AM). Evening collection times were at the end of shift, or 12 hours later. Overall, cortisol levels were significantly higher on the first day than on the second day for both groups. One reason for this result is that the participants reported experiencing stress before returning to work after having been off for several days. That is, some nurses noted that the anticipation of returning to work after several days off can be stressful, whereas the last shift can be less stressful because of looking forward to time off.

The results of our study are similar to the results of other studies of nurses that reported a positive relationship between increasing stress over consecutive shifts varying from 8 to 12 hours. ${ }^{[20,21]}$ However, it is difficult to assess consecutive shifts accurately owing to a lack of administrative data and because of issues related to schedule changes. ${ }^{[30]}$

Several states and nursing professional organizations are developing staffing standards, ${ }^{[31]}$ although mandatory overtime regulations vary from state to state. ${ }^{[14]}$ Individual lifestyle, the culture of the unit, and organizational policies can influence the intensity of work-related fatigue and may moderate the relationship between work stress and fatigue. ${ }^{[1]}$

\section{Strengths and limitations}

The generalizability of our study results are limited for several reasons. One is that the nurses in this study worked at a Magnet-designated hospital, making this study representa-

Published by Sciedu Press tive of the top $10 \%$ of high-performing acute care hospitals nationally. ${ }^{[15]}$ The nurse-to-patient ratio was 1 nurse to 4 to 5 patients on day shift, which is an ideal nurse-to-patient ratio in a medical/surgical unit. The use of a convenience sample also limited generalizing to medical/surgical nurses and nurse leaders at large. Although the small sample size was adequate to achieve significance, that the sample size was less than 60 subjects resulted in a small sample of nurse leaders $(n=16)$. In addition, the study evaluated only nurses in nonrotating 12-hour dayshift. To be included, the participants had to have been off duty for at least 2 days before the collection of baseline data. In addition, a limitation of this study was the menstrual cycles of the nurses were not evaluated.

Limitations included salivary samples sometimes were not collected at the end of shift, mainly as the result of overtime work. This reduced the sample size to $54 \mathrm{AM}$ and $53 \mathrm{PM}$ collections. The self-reporting of perceived stress was between 30 minutes to 12 hours prior to beginning the initial 12-hour shift. Additionally, the measure for work-related stress may not have been valid for nurse leaders because the NSS is weighted heavily toward patient care tasks.

To obtain consistent results, the collection protocol was identical for all subjects. Sampling guidelines and scheduling were followed to reduce risk of preanalytic errors. To our knowledge, no prior studies in the United States have used 12-hour day shift nurses over 2 consecutive shifts in stressbiomarker research, in which a repeated-measures design was followed for evaluating perceived stress, fatigue, and cortisol levels. 


\section{Conclusion}

Nurses from direct care staff to executives should be educated in and demonstrate best practices in relation to ANA endorsements on fatigue and interventions to lessen the risk. ${ }^{[32]}$ Nurses need to inspire and encourage self-care within their practice. The adaptation of ANA interventions to decrease fatigue reduces the risk of patient errors and fosters a healthy work environment. Researchers have recommended work hour regulations within the hospital to minimize the effects of fatigue. ${ }^{[13]}$ For instance, Hazzard et al. ${ }^{[33]}$ recommended limiting the workweek to no more than 3 consecutive 12-hour shifts. Competing initiatives pressure nurse leaders to work long hours to support organizational goals, sometimes at the expense of a healthy work environment. According to ANA recommendations, nurse leaders should minimize long-hour days in their schedules, limit consecutive shifts, and support a healthy lifestyle that includes exercise, healthy eating, and time off between shifts to foster adequate sleep. ${ }^{[33]}$ Leaders should be knowledgeable of stress factors in their nurses that potentiate decrements in personal health. ${ }^{[34]}$ In addition, leaders should be keenly aware of ineffective coping, passive behavior, and negative communication associated with fatigue. ${ }^{[12]}$ Employee Assistance Programs are available to help leaders provide support to nurses during stressful times and support fatigue countermeasure interventions. ${ }^{[34]}$

Nurses should be knowledgeable of ANA's principles of Safe Nurse Staffing, which outline organizational guidelines and good decision-making practices. ${ }^{[12]}$ In addition, the ANA website (www.nursingworld.org) offers the Healthy Nurse program, which provides information on sleep, weight control, fatigue, and other health-related topics to help nurses adjust to shiftwork. Although nurses are generally aware of the attitudes and practices to maintain a healthy work environment, often these practices are not applied toward self-care. ${ }^{[35]}$ Potential interventions to reduce fatigue include providing adequate time between patient admissions, establishing a reasonable workload, implementing various work break strategies, and accessing help from other coworkers. ${ }^{[33]}$

\section{Recommendations for future research}

Future studies should focus on the relationships of stress, other biomarkers, and fatigue over extended consecutive shifts to include three or more consecutive days worked which is common in hospital-based nursing practice. This study did not represent overtime work hours beyond 12.5 hours or more than 2 consecutive shifts or night shift nurses. The threshold of when fatigue degrades performance needs to be established in longitudinal studies. Research should expand outcome measures to include the effect of fatigue on specific cognitive functioning tasks, error rates, and nursesensitive outcomes. Altered work environment variables, such as various work break interventions, specific scheduling patterns, and workload of patient care need to be evaluated for effectiveness in reducing fatigue. Lastly, recognizing the potential moderating effect of taking time off, incorporating one's belief system into practice, and social support provided through strong work and family relationships should continue to be evaluated for effective management of stress and fatigue. ${ }^{[16]}$

While nurses' perceived stress and fatigue increased over 2 consecutive 12-hour workdays, contrary to our hypothesis, cortisol decreased from Day 1 to Day 2. Nurse leaders and nurses reported similar stress, fatigue, and cortisol levels despite dissimilar job responsibilities. At the start of the work schedule, mean stress and fatigue scores were significantly higher in medical/surgical nurses than in nurse leaders. Cortisol levels were similar between the 2 groups, until the last collection, when cortisol levels were significantly higher $(p=.001)$ in medical/surgical nurses than in nurse leaders. Stress and fatigue scores quickly increased in nurse leaders to match those of the nurses by the end of day 2. Our findings support using Kang's biobehavioral approach to stress research by further investigating the relationship of cortisol and other biomarkers as measures of the body's response to stress. $^{[6]}$

\section{ACKnOWLedgements}

This study was funded by Houston Methodist Willowbrook Hospital, Center for Professional Excellence. We acknowledge Jennifer Holmes, ELS, for editorial support as a freelance medical writer.

\section{CONFlicts OF InTEREST Disclosure}

The authors declare that there is no conflict of interest.

\section{REFERENCES}

[1] Smith-Miller CA, Shaw-Kokot J, Curro B, et al. An integrative review: fatigue among nurses in acute care setting. J Nurs Adm. 2014; 44(9): 487-494. https ://doi.org/10.1097/NNA.0000000000 000104
[2] Geiger-Brown J, Rogers VE, Trinkoff AM, et al. Sleep, sleepiness, fatigue, and performance of 12-hour-shift nurses. Chronobiol Int 2012; 29(2): 211-219. https://doi.org/10.3109/07420528.2 011.645752

[3] Johnson AL, Jung L, Brown KC, et al. Sleep deprivation and errors 
in nursing who work night shift. J Nurs Adm. 2014; 44(1): 17-22. https://doi.org/10.1097/NNA.0000000000000016

[4] Olds DM, Clarke SP. The effect of work hours on adverse events and errors in health care. J Safety Res. 2010; 41(2): 153-162. https://doi.org/10.1016/j.jsr.2010.02.002

[5] Bae SH, Trinkoff A, Jing H, et al. Factors associated with hospital staff nurses working on-call hours. Workplace Health Saf. 2013; 61: 203-211. https://doi .org/10.1177/216507991306100504

[6] Kang D, Rice M, Park N, et al. Stress and inflammation: a behavioral approach for nursing research. West J Nurs Res. 2010; 32(6): 730-760. https://doi.org/10.1177/0193945909356556

[7] Almadi T, Cathers I, Chow CM. Associations among work-related stress, cortisol, inflammation, and metabolic syndrome. Psychophysiology. 2013; 50(9): 821-830. https://doi.org/10.1111/psyp .12069

[8] Leka S, Jain A. Health impact of psychosocial hazards at work: an overview [Internet]. World Health Organization. 2010. Available from: http://whqlibdoc.who.int/publication/2010 /9789241500272_eng.pdf

[9] Barker LM, Nussbaum MA. Fatigue, performance and the work environment: A survey of registered nurses. J Adv Nurs. 2011; 67(6): 1370-1382. https ://doi.org/10.1111/j.1365-2648.2010.0 $5597 . x$

[10] Chen J, Daraisehn NM, Davis KG, et al. Sources of work-related acute fatigue in the United States hospital nurses. Nurs Health Sci. 2014; 16(1): 19-25. https://doi.org/10.1111/nhs.12104

[11] Rice V. Stress and the workplace. In: Handbook of Stress, Coping and Health: Implications for Nursing Research, Theory and Practice. 2nd ed. Thousand Oaks (CA): SAGE; 2011.

[12] American Nurses Association. Executive summary: Health risk appraisal (HRSA) preliminary findings [Internet]. Silver Spring (MD): ANA; 2015. Available from: http://www. nursingworld.org/H RA-Executive-Summary

[13] Bae S, Fabry D. Assessing the relationships between nurse work hours/overtime and nurse and patient outcomes: systematic literature review. Nurs Outlook. 2014; 62(2): 138-156. https://doi.org/ $10.1016 / j$. outlook. 2013.10.009

[14] Bae S. Presence of nurse mandatory overtime regulations and nurse and patient outcomes. Nurs Econ. 2013; 31: 59-68. PMid:23691746

[15] American Nurses Credentialing Center. Forces of magnetism [Internet]. Silver Spring (MD): ANCC; 2015. Available from: http: //www . nursecredentialing.org/ForcesofMagnetism.aspx

[16] Estryn-Behar M, Van der Heijden BIJM; NEXT study group. Effects of extended work shifts on employee fatigue, health, satisfaction, work/family balance, and patient safety. Work. 2012; 41: 4283-4290. PMid:22317378 https://doi .org/10.3233/WOR-2 012-0724-4283

[17] US Department of Health and Human Services, Health Resources and Services Administration. The U.S. nursing workforce: Trends in supply and education results in brief [Internet]. Washington (DC): HRSA; 2013 Apr. Available from: http://bhpr.hrsa.gov/heal thworkf orce/supplydemand/nursing/nursingworkforce/

[18] De Castro AB, Fujishiro K, Rue T, et al. Association between work schedule characteristics and occupational injury and illnesss. Int Nurs Rev. 2010; 57(2): 188-194. https://doi.org/10.1111/j.1466 $-7657.2009 .00793 . \mathrm{x}$

[19] National Institute of Nursing Research. NINR Strategic Plan. Bethesda (MD): National Institute of Nursing Research; 2011.

[20] Chang YS, Wu YH, Su SF, et al. Factors related with the ability to maintain wakefulness in the daytime after fast and forward rotating shifts. Kaohsiung J Med Sci. 2013; 29(11): 611-616. https://doi.org/10.1016/j.kjms.2013.01.012

[21] Chang Y, Chen H, Wu Y, et al. Rotating night shifts too quickly may cause anxiety and decreased attentional performance, and impact prolactin levels during the subsequent day: A case control study BMC Psychiatry. 2014; 14(1): 218-230. https ://doi .org/10.1 186/s12888-014-0218-7

[22] Han K, Trinkoff AM, Geiger-Brown J. Factors associated with work-related fatigue and recovery in hospital nurses working 12hour shifts. Workplace Health Saf. 2014; 62(10): 409-414. https : //doi .org/10.3928/21650799-20140826-01

[23] Lin P, Chen C, Pan S, et al. The association between rotating shift work and increased occupational stress in nurses. J Occup Health. 2015; 57(4): 307-315. https : //doi .org/10.1539/joh.13-028 $4-0 \mathrm{~A}$

[24] Niu SF, Chung MH, Chen $\mathrm{CH}$, et al. The effect of shift rotation on employee cortisol profile, sleep quality, fatigue and attention level: a systematic review. J Nurs Res. 2011; 19(1): 68-81. https://doi.org/10.1097/JNR.0b013e31820c1879

[25] Karhula K, Harma M, Sallinen M, et al. Association of job strain with cortisol and alpha-amylase among shift-working health care professionals in laboratory and field. Biol Res Nurs. 2016; 18(1): 101-112. https://doi.org/10.1177/1099800415577801

[26] An K, Starkweather A, Sturgill J, et al. State of the science: salivary biomarker utilization for stress research. Perspect Nurs Sci. 2014; 11(2): 87-93. https://doi.org/10.16952/pns.2014.11.2.8 7

[27] Dittner AJ, Wessely SC, Brown RG. The assessment of fatigue: a practical guide for clinicians and researchers. J Psychosom Res. 2004; 56(2): 157-170. https ://doi.org/10.1016/S0022-399 9 (03) 00371-4

[28] Gray-Toft P, Anderson JG. The Nursing Stress Scale: development of an instrument. J Behav Assess. 1981; 3(1): 11-23. https : //doi.org/10.1007/BF01321348

[29] Cohen S, Janicki-Deverts D, Miller GE. Psychological stress and disease. JAMA. 2007; 298(14): 1685-1687. https ://doi .org/10 $.1001 /$ jama. 298.14.1685

[30] Hopcia K, Dennerlein JT, Hashimoto D, et al. A case-control study of occupational injuries for consecutive and cumulative shifts among hospital registered nurses and patient care associates. Workplace Health Saf. 2012; 60: 437-444. https://doi.org/10.3928/21 650799-20120917-39

[31] Reed K. Promoting healthy work hours for nurses. Nursing. 2013; 43(1): 64-65. https://doi.org/10.1097/01. NURSE.0000422 658.39909. 37

[32] American Nurses Association. Nurse fatigue [Internet]. Silver Spring (MD): ANA; 2014. Available from: https://www. nursingworld .org/fatigue

[33] Hazzard B, Johnson K, Dordunoo D, et al. Work-and nonorganizational factors associated with PACU nurses fatigue. J Post Anesth Nurs. 2013; 28: 201-209. https://doi.org/10.1016/j .jopan. 2012.06.010

[34] American Nurses Association. ANA calls for stronger collaboration between RNs, employers to reduce risks from nurse fatigue [Internet]. Silver Spring (MD): ANA; 2014. Available from: http: //www. nursingworld.org/FunctionalMenuCategories/Me diaResources/PressReleases/2014-PR/Stronger-Colla boration-to-Reduce-Risks-from-Nurse-Fatigue.html

[35] Nahm ES, Warren J, Zhu S, et al. Nurses' self-care behaviors related to weight and stress. Nursing Outlook. 2012; 60(5): e23-31. 